\title{
USO DEL MODELO AMMI PARA EL ANÁLISIS DE LA INTERACCIÓN GENOTIPO AMBIENTE EN VARIEDADES DE MAÍZ AMILÁCEO DE TAYACAJA, PERÚ
}

\section{Ammi model for genotype by environment interaction analysis in starchy corn from Tayacaja, Peru}

\author{
iD Pedro José García Mendoza' iD Darío Emiliano Medina Castro² iD Gino Paul Prieto Rosales ${ }^{2}$ \\ (iD) Damián Manayay Sánchez ${ }^{3}$ (iD Ronald Ortecho Llanos ${ }^{1}$ \\ 'Universidad Nacional Autónoma de Tayacaja Daniel Hernández Morillo, Perú \\ ${ }^{2}$ Universidad Nacional de Trujillo, Perú. \\ ${ }^{3}$ Universidad Nacional del Santa, Perú. \\ Correspondencia: \\ Dr. Pedro José García Mendoza \\ pedrogarcía@unat.edu.pe
}

\section{RESUMEN}

La interacción genotipo por ambiente (IGA) resulta la principal limitante para seleccionar los mejores genotipos para diversos ambientes. El objetivo de este estudio fue utilizar el modelo de efectos principales aditivos e interacción multiplicativa (AMMI) para evaluar la IGA de 25 variedades de maíz amiláceo. La información fue generada en cuatro ensayos establecidos en ambientes contrastantes de la provincia de Tayacaja, Perú, en el ciclo del cultivo 2019 - 2020. Se utilizó el diseño alfa látice 5×5, con tres repeticiones, en donde las unidades experimentales estuvieron constituidas por dos hileras de $4 \mathrm{~m}$ de longitud, con arreglos espaciales de 0,80 m entre hileras y 0,20 m entre puntos de siembra. La IGA se midió a través del rendimiento de grano, ajustado a $15 \%$ de humedad. Una vez comprobada la importancia de la IGA en los experimentos, se realizó el análisis multivariado, para obtener los valores singulares de los términos AMMI que resultaron significativos para genotipos y ambientes. La IGA resultó altamente significativa y explicó alrededor del $33 \%$ de la variación fenotípica del rendimiento. El modelo AMMI explicó alrededor del 92 \% de la variación debida a la IGA, en donde los dos primeros ejes concentraron toda esta variación y permitieron identificar variedades con adaptación específica y otras con amplia adaptación a los ambientes de prueba. Los resultados sugieren que el modelo AMMI fue apropiado para evaluar la IGA y para identificar las mejores variedades para cada ambiente de prueba.

Palabras clave: Interacción genotipo ambiente, Zea mays L., adaptabilidad, rendimiento, potencial productivo. 


\begin{abstract}
The main limitation for selecting the best genotypes for different environments is the genotype by environment interaction (GAl). The objective of this study was to use the additive main effects and multiplicative interaction (AMMI) model to evaluate the GAI of 25 starchy corn varieties. Four trials established in contrasting environments in the province of Tayacaja, Peru, 2019-2020 crop cycle were used to generate information. A $5 \times 5$ alpha lattice design with three replications was used, where the experimental units consisted of two rows, $4 \mathrm{~m}$ long, with spatial arrangements of $0.80 \mathrm{~m}$ between rows and $0.20 \mathrm{~m}$ between planting points. GAl was measured through grain yield, adjusted to $15 \%$ humidity. Once the importance of GAl in trials was verified, a multivariate analysis was performed to obtain the singular values of the significant AMMI terms for genotypes and environments. The GAl was highly significant and explained about $33 \%$ of the phenotypic variation in performance. The AMMI model explained around $92 \%$ of the variation due to the GAl, where the first two axes concentrated all this variation and allowed to identify varieties with specific adaptation and others with broad adaptation to the test environments. The results suggested that the AMMI model was appropriate for evaluating GAI and for identifying the best varieties for each test environment.
\end{abstract}

Keywords: Genotype by environment interaction, Zea mays L., adaptability, yield, productivity potential

\section{INTRODUCCIÓN}

En un programa de mejoramiento genético la fase considerada de mayor complejidad es la relacionada con la evaluación de los genotipos experimentales, debido a la necesidad de evaluar un gran número de genotipos en el mayor número de ambientes posibles, lo que además la convierte en la etapa que demanda la mayor disponibilidad de recursos (Seyoum et al., 2019; Souza et al., 2009). La complejidad se debe a que la interacción genotipo por ambiente (IGA) dificulta la selección de los mejores genotipos para todos los ambientes de prueba. Por esta razón, la IGA es el factor que determina la extensión de las pruebas y por consiguiente sus costos en un programa de mejoramiento genético ( $L i$ et al., 2020). Sin embargo, a pesar de la importancia del estudio de la IGA, incluyendo la adaptabilidad y estabilidad de los materiales genéticos evaluados, generalmente las recomendaciones de los mejores genotipos a seleccionar se basan en el desempeño medio de éstos a través de los ambientes (Adnan et al., 2020). No obstante, este criterio para realizar las recomendaciones no es el más apropiado, puesto que bajo estas condiciones la IGA no permite identificar de manera clara a los genotipos superiores (Adnan et al., 2020; Souza et al., 2009).
La IGA, definida como la respuesta diferencial en el comportamiento de dos o más genotipos evaluados en diferentes ambientes (Blum, 1988; Bowman, 1972; García et al., 2020), es considerada como un fenómeno universal, sobre todo, cuando se prueban diferentes genotipos en diversos ambientes, siendo un tema relevante para mejoradores de plantas y agrónomos, cuyo interés principal es determinar el comportamiento agronómico de cultivares evaluados en diferentes ambientes, que faciliten la recomendación de los mejores genotipos con mayor posibilidad de éxito en las regiones específicas donde se practica la producción comercial del cultivo (García et al., 2020). En consecuencia, la IGA representa el mayor reto que enfrentan los agrónomos y mejoradores de plantas, para recomendar los mejores genotipos para un rango de diferentes ambientes o para ambientes específicos de interés (Mudada et al., 2017).

Cuando se tiene un conjunto de variedades que pueden ser sembradas por pequeños agricultores ubicados en regiones con marcadas diferencias agroecológicas, previo a recomendarles la variedad ideal para las diferentes regiones de 
producción, es esencial conocer la contribución del efecto de la IGA sobre la prospectiva variedad. Esto significa que cuando un grupo de genotipos son probados en ambientes contrastantes, se podrían identificar algunos de ellos con amplia adaptación a los diversos ambientes de prueba, mientras que otros podrían mostrar adaptación a condiciones de ambientes específicas (Chimonyo et al., 2014).

Un análisis de la varianza (ANOVA) combinado puede utilizarse para cuantificar el efecto de la IGA y describir los efectos principales de genotipos y ambientes (Setimela et al., 2018). No obstante, el ANOVA, por sí solo, no puede explicar la totalidad del efecto de la IGA, de tal forma que se puedan identificar los genotipos con amplia adaptación o con adaptación específica (Admassu et al., 2008). La mejor alternativa para explicar la interacción de los efectos principales más allá del ANOVA, es utilizando otros modelos estadísticos, tal como lo sugerido por Paz et al. (2018).

Diversos métodos estadísticos multivariados han sido desarrollados para analizar e interpretar la IGA, siendo uno de ellos el modelo de efectos principales aditivos e interacción multiplicativa, AMMI, propuesto por Gauch y Zobel (1988) y usado frecuentemente en la actualidad por muchos autores en diversos trabajos (Adnan et al., 2020; Mudada et al., 2017; Rusinamhodzi et al., 2020). El método AMMI utiliza el análisis de varianza (ANOVA), seguido del análisis de componentes principales aplicado a la suma de cuadrados, que, de acuerdo al ANOVA, le corresponden a la interacción genotipo $x$ ambiente. Se basa en un modelo estadístico lineal-bilineal (Crossa et al., 2000), en el que los efectos principales de genotipos y de ambientes, considerados términos lineales, se explican mediante un análisis de varianza convencional, mientras que el componente bilineal (no aditivo) se le atribuye a la interacción genotipo $\times$ ambiente y se analiza mediante la técnica de componentes principales (Falcon et al., 2020; García et al., 2020; Ngaboyisonga et al., 2016; Paz et al., 2018; Rusinamhodzi et al., 2020). La metodología de análisis y los principios estadísticos y científicos que sustentan este método han sido detallados en el pasado en trabajos previos (Gauch y Zobel., 1996; Vargas y Crossa., 2000; Yan et al., 2007). La fortaleza de este método radica en la posibilidad de hacer una representación gráfica (biplot) de la variabilidad de las observaciones, considerando genotipos y ambientes (Crossa et al., 2000; Gabriel, 1971), lo cual permite visualizar fácilmente los genotipos con mejores posibilidades de éxito para un rango variable de ambientes o para ambientes específicos. No obstante, esto sólo se considera apropiado si los dos primeros componentes principales explican una parte importante de la variabilidad correspondiente a la matriz de la IGA (Falcon et al., 2020; Gauch y Zobel., 1988; Setimela et al., 2018). El primer componente representa a los efectos repetibles de la IGA y el segundo a los efectos sin patrón definido o ruido; de esta manera, el método conduce a la identificación de genotipos estables o con adaptación específica (Crossa, 1990; García et al., 2020).

En el Perú han sido pocos los trabajos de investigación referidos al estudio de la IGA y de los publicados a la fecha en el cultivo de maíz (Chura-Chuquija y Huanuqueño-Coca, 2015; López-Morales et al., 2019); ninguno se ha realizado en la región de la sierra. De estos estudios, sólo uno (López-Morales et al., 2019), ha utilizado el método AMMI, lo cual se presume se deba a varios factores, dentro de los cuales se pueden destacar la necesidad de disponer de la logística y recursos económicos apropiados y contar con un equipo técnico capacitado y dispuesto a desarrollar los experimentos en condiciones de ambientes de difícil acceso, que permita evaluar un gran número de genotipos en diversos ambientes contrastantes. En este país, igual que en el resto de Latinoamérica, el mayor énfasis se ha dado al desarrollo de cultivares de maíz para las regiones que representan las mayores áreas de siembra, posiblemente debido al mayor interés económico que estas áreas representan para las empresas de semillas que operan en la región.

Desde mediados del año 2018, la Universidad Nacional Autónoma de Tayacaja, "Daniel Hernández Morillo" (UNAT), viene desarrollando 
un proyecto de investigación agrícola en maíz, cuyo principal objetivo es seleccionary desarrollar variedades de maíz con elevado potencial genético de producción y calidad culinaria e industrial, que contribuya a mejorar la rentabilidad de toda la cadena agro productiva vinculada al cultivo de maíz amiláceo en la Provincia de Tayacaja (UNAT, 2018). Dicho proyecto, ha contemplado la evaluación de un grupo de variedades de maíz amiláceo recolectadas en diversos ambientes contrastantes de la región, en donde se ha podido obtener información nueva y relevante para orientar a los agricultores en la selección de las mejores variedades para las diferentes áreas agroecológicas de la provincia. De esta forma, el objetivo principal de este estudio fue utilizar el modelo AMMI para evaluar la interacción genotipo por ambiente de variedades de maíz amiláceo establecidas en diferentes ambientes de la provincia, a fin de facilitar la selección de los materiales más aptos para los diferentes ambientes productores de maíz amiláceo de la provincia de Tayacaja, en el ciclo del cultivo 2019 - 2020.

\section{MATERIALES Y MÉTODOS \\ Material experimental y ambientes de evaluación}

Los experimentos estuvieron conformados por 22 variedades experimentales de maíz amiláceo recolectadas en la provincia de Tayacaja, más 3 cultivares proporcionados por el Instituto Nacional de Innovación Agraria (INIA) Cajamarca, utilizadas como testigos (Tabla 1). El grupo de variedades experimentales y testigos incluyó maíces amiláceos del tipo Astilla, Carhuay, Pisturunto y Chullpi, utilizados principalmente como cancha, del tipo cusqueado, para mote y choclo y maíces morados, utilizados para chicha y como fuentes de antocianinas.

Los ensayos fueron establecidos en 4 localidades, representativas de ambientes contrastantes, distribuidas en diferentes distritos productores de maíz amiláceo de la provincia de Tayacaja, Huancavelica, Perú, en el ciclo del cultivo 2019 2020 (Tabla 2).

\section{Diseño experimental y análisis estadístico}

Se utilizó un diseño experimental de bloques incompletos, Alfa Látice $5 \times 5$, con tres repeticiones. La unidad experimental estuvo integrada por dos hileras de $4 \mathrm{~m}$ de longitud, utilizando arreglos espaciales de 0,80 m de separación entre hileras y 0,20 m entre puntos de siembra; los experimentos fueron diseñados para colocar dos semillas por punto de siembra (84 semillas por parcela) para posteriormente realizar un raleo, transcurridos 30 días después de la siembra del experimento, dejando sólo una planta por punto de siembra, previendo una población final de 62500 plantas ha ${ }^{-1}$, aproximadamente. 
Tabla 1

Nombres y lugares de origen de las variedades de maíz amiláceo incluidas en el estudio en el ciclo del cultivo 2019-2020

\begin{tabular}{|c|c|c|c|c|}
\hline Entrada & Ecotipo -Variedad & Código & Localidad & Distrito \\
\hline $\begin{array}{l}1 \\
2\end{array}$ & $\begin{array}{l}\text { Astilla Amarilla-DH } \\
\text { Astilla Blanca-DH }\end{array}$ & $\begin{array}{l}\mathrm{G} \\
\mathrm{G}\end{array}$ & Los Ángeles de Cucharán & $\begin{array}{c}\text { Daniel } \\
\text { Hernández }\end{array}$ \\
\hline 3 & Carhuay-Run & $\mathrm{G}_{3}$ & Rundo & $\begin{array}{c}\text { Daniel } \\
\text { Hernández } \\
\end{array}$ \\
\hline 4 & Carhuay-Q & $\mathrm{G}$ & Anjara Pata & \multirow{3}{*}{ Quishuar } \\
\hline 5 & Bolón-Q & $\mathrm{G}_{s}$ & Pachas & \\
\hline 6 & Chullpi-Q & $\mathrm{G}$ & Yacutoclla & \\
\hline 7 & Astilla Blanca-P & $\mathrm{G}$ & Ahuayta Casay & \multirow{4}{*}{ Pampas } \\
\hline 8 & Cusqueado-P & $\mathrm{G}_{s}$ & Gentil Pampas & \\
\hline 9 & Carhuay-P & $\mathrm{G}_{0}$ & Mantacra & \\
\hline 10 & Blanco Gigante-P & $G_{10}$ & Providencia Mantacra & \\
\hline 11 & Astilla Blanca-C & $\mathrm{G}_{n}$ & $\begin{array}{c}\text { Agua Dulce, Com. } \\
\text { Campesina San Julián Alto }\end{array}$ & Colcabamba \\
\hline 12 & Almidón-ST & $\mathrm{G}_{12}$ & Buenos Aires & \multirow{3}{*}{$\begin{array}{l}\text { Santiago de } \\
\text { Tucuma }\end{array}$} \\
\hline 13 & Astilla Blanca-ST & $\mathrm{G}_{13}$ & Huayrapire & \\
\hline 14 & Chullpi-ST & $\mathrm{G}_{*}$ & Usnupampa & \\
\hline $\begin{array}{l}15 \\
16 \\
\end{array}$ & $\begin{array}{l}\text { Pistiada-Pi } \\
\text { Carhuay-Pi }\end{array}$ & $\begin{array}{l}G_{16} \\
G_{16}\end{array}$ & Muyupata & Pichos \\
\hline $\begin{array}{l}17 \\
18 \\
\end{array}$ & $\begin{array}{c}\text { Morocho Duro-Pi } \\
\text { Morocho Rojo Duro-Pi }\end{array}$ & $\mathrm{G}_{11}$ & Chinchipampa & Pichos \\
\hline 19 & Paru Rojo-Pi & $G_{10}$ & Willcanpata & \multirow{4}{*}{ Pichos } \\
\hline 20 & Maíz Morado-Pi & $\mathrm{G}_{\infty}$ & Huaychaocruz & \\
\hline 21 & Paru Occe-Pi & $\mathrm{G}_{21}$ & Sheccha & \\
\hline 22 & Huamamsara-Pi & $\mathrm{G}_{n}$ & Huasahuayoco & \\
\hline 23 & Choclero-101 & $\mathrm{T}_{\mathrm{T}}$ & \multirow{3}{*}{ INIA Cajamarca } & \multirow{3}{*}{ Testigos } \\
\hline 24 & Choclero-INIA-603 & $\mathrm{T}_{2}$ & & \\
\hline 25 & INIA-601 & $T_{0}$ & & \\
\hline
\end{tabular}


Para determinar el efecto de interacción genotipo por ambiente en las variedades estudiadas, se utilizó el rendimiento de grano (RG), ajustado a $15 \%$ de humedad, expresado en $t$ ha $^{-1}$. Los ensayos fueron establecidos y cosechados en forma manual y el manejo agronómico se realizó conforme a las recomendaciones técnicas establecidas para el cultivo en cada ambiente de prueba.

Se realizó un análisis de varianza (ANOVA) individual y combinado, utilizando la media de cada unidad experimental, mediante el procedimiento GLM del SAS (SAS, 2003).

Tabla 2

Localidades y fechas de siembra y de cosecha de los experimentos

\begin{tabular}{|c|c|c|c|c|c|c|}
\hline \multirow{2}{*}{$\mathrm{N}^{\circ}$} & \multirow{2}{*}{$\begin{array}{l}\text { Nombre de } \\
\text { Localidad }\end{array}$} & \multirow{2}{*}{ Ubicación política } & \multicolumn{2}{|c|}{ Ubicación geográfica } & \multirow{2}{*}{ msnm } & \multirow{2}{*}{$\begin{array}{l}\text { Fecha de } \\
\text { siembra y } \\
\text { de cosecha }\end{array}$} \\
\hline & & & Latitud & Longitud & & \\
\hline 1 & Beto Ega & $\begin{array}{l}\text { Finca Sr. Adalberto } \\
\text { Ega, Anexo Rundo, } \\
\text { Distrito Daniel } \\
\text { Hernández }\end{array}$ & $12^{\circ} 21^{\prime} 29.7^{\prime \prime} \mathrm{S}$ & $74^{\circ} 50^{\prime} 30.3^{\prime \prime} \mathrm{W}$ & 3217 & $\begin{array}{l}\text { 23/10/2019; } \\
09 / 06 / 2020\end{array}$ \\
\hline 2 & El Rosario & $\begin{array}{c}\text { Finca del Sr. Clímaco } \\
\text { Culcapuza, Centro } \\
\text { Poblado Mantacra, } \\
\text { Distrito Pampas }\end{array}$ & $12^{\circ} 29^{\prime} 37.4^{\prime \prime} \mathrm{S}$ & $74^{\circ} 49^{\prime} 43.2^{\prime \prime} \mathrm{W}$ & 2860 & $\begin{array}{l}\text { 22/10/2019; } \\
05 / 06 / 2020\end{array}$ \\
\hline 3 & Porvenir & $\begin{array}{c}\text { Finca del Sr. Roberto } \\
\text { Palomino Espinoza } \\
\text { Poblado Porvenir, } \\
\text { Distrito Pampas }\end{array}$ & $12^{\circ} 28^{\prime} 51.69^{\prime \prime S}$ & $74^{\circ} 49^{\prime} 43.17^{\prime \prime} \mathrm{W}$ & 3143 & $\begin{array}{l}30 / 10 / 2019 \\
12 / 06 / 2020\end{array}$ \\
\hline 4 & Colcabama & $\begin{array}{c}\text { Finca del Instituto } \\
\text { Tecnológico, Distrito } \\
\text { Colcabamba }\end{array}$ & $12^{\circ} 24^{\prime} 24.41^{\prime \prime S}$ & $74^{\circ} 40^{\prime} 55.95^{\prime \prime} \mathrm{W}$ & 3039 & $\begin{array}{l}\text { 04/12/2019; } \\
10 / 07 / 2020\end{array}$ \\
\hline
\end{tabular}

El ANOVA combinado se efectuó conforme al siguiente modelo matemático: $Y_{i j k l}=\mu+t_{i}+$ $R_{j(l)}+\beta_{k(j)}+l_{1}+t l_{i l}+\epsilon_{i j k l^{\prime}}$ donde $Y_{i j k l}$ representó a la observación obtenida en el i-ésimo genotipo, evaluado en la j-ésima repetición dentro de la I-ésima localidad, en el k-ésimo bloque dentro de la j-ésima repetición y en la l-ésima localidad; $\mu$ es la media general; $t_{i}$ representó al efecto fijo del i-ésimo genotipo utilizado en los ensayos, con i $=1,2, \ldots, 25 ; R_{j(l)}$ fue el efecto aleatorio de la j-ésima repetición dentro de la l-ésima localidad, con $j=1,2,3 ; \beta_{k(j))^{\prime}}$ fue el efecto aleatorio del k-ésimo bloque dentro de la j-ésima repetición y la l-ésima localidad, con k=1,2, ., 5; $I_{1}$ representó el efecto aleatorio de la l-ésima localidad, con I= 1,2 y $4 ; t l_{i l}$ fue el efecto aleatorio de la interacción entre el i-ésimo genotipo con la l-ésima localidad; $\epsilon_{i j k l}$ representó el error asociado a la observación $Y_{i j k l^{\prime}}$ conforme a (García et al., 2020; Steel y Torrie, 1988).
Para la comparación de medias se construyó un intervalo de confianza para la media general, utilizando la siguiente expresión matemática, conforma a Steel y Torrie (1988).

$\underline{X}-Z \frac{\sigma}{\sqrt{n}} \leq \mu \leq \underline{X}+Z \frac{\sigma}{\sqrt{n}}$,

donde: $\underline{X}$ representa a la media muestral; $Z$ es el valor de $\boldsymbol{Z}$ tabulado para un nivel de confianza del 5\%, $\sigma$ representa a la desviación estándar de la población; $\mu$ es la media general poblacional y $n$ es el número de observaciones utilizadas para la estimación de la media muestral.

También se realizaron contrastes ortogonales para determinar las diferencias entre los materiales locales y las variedades utilizadas como testigos.

\section{Modelo AMMI}

Comprobada la presencia de la IGA en el ANOVA 
combinado, se procedió a realizar el análisis multivariado de componentes principales, para obtener los valores singulares de los términos AMMI que dieron significancias para los genotipos y ambientes, conforme a lo establecido por varios autores (Falcon et al., 2020; Ngaboyisonga et al., 2016; Vargas y Crossa., 2000), utilizando sólo las medias ajustadas del rendimiento de grano, de acuerdo al siguiente modelo matemático:

$Y_{i j}=\mu+g_{i}+a_{j}+\sum_{k=1}^{n} \lambda_{k} \gamma_{i k} \alpha_{j k}+\rho_{i j}+\xi_{i j}$, donde $Y_{i j}$ representó la media del i-ésimo genotipo en el j-ésimo ambiente; $\mu$ es la media general; $g_{i}$ y $a_{j}$ son los efectos del i-ésimo genotipo y j-ésimo ambiente, respectivamente; n es el número de componentes principales (CP) retenidos en el modelo; $k$ es el valor singular para cada CP; ik son valores de los vectores de los genotipos para cada CP; $j k$ son los valores de los vectores de los ambientes para cada CP; ij es el residual de la IGA y $\varepsilon_{i j}$ es el error experimental medio. Los valores genotípicos y ambientales del modelo utilizado fueron obtenidos por medio del procedimiento de lenguaje interactivo de matrices del SAS (PROC IML) (SAS, 2003). La significancia de cada CP fue medida mediante una prueba de $\mathrm{F}$ aproximada al nivel 0,05 de probabilidad, comparando el cuadrado medio de cada CP, con el cuadrado medio del error experimental, conforme a Crossa et al. (1990). La construcción del gráfico de doble representación biplot del modelo AMMI se hizo conforme a lo reportado por Chimonyo et al. (2014) y García et al. (2009).

\section{RESULTADOS Y DISCUSIÓN}

El análisis de varianza combinado determinó que las variedades de maíz amiláceo (VMA) no mantuvieron sus niveles de productividad en los diferentes ambientes de evaluación, observándose diferencias estadísticamente importantes $(p<0,01)$ para el efecto de interacción genotipo x ambiente (IGA) (Var*Amb) (Tabla 3). Estos resultados sugieren que el rendimiento de grano mostrado por las variedades, varió en función de la condición ambiental presente en cada localidad donde fueron evaluadas; también se encontraron diferencias estadísticas significativas entre los ambientes de prueba $(p<0,05)$ y para el efecto de variedades $(p<0,01)$, lo que sugiere las condiciones contrastantes de los ambientes de prueba y la divergencia genética entre las variedades utilizadas en el estudio. En este estudio, la IGA explicó alrededor del 32.51 \% de la variación fenotípica del rendimiento de las variedades de maíz amiláceo. Falcón et al. (2020), encontraron que la IGA explicó entre 9.0 y 20.4 $\%$ de la varianza fenotípica, con mayores efectos para los componentes del rendimiento.

El análisis de varianza individual también detectó diferencias estadísticas significativas $(p<0,01)$ para el efecto de variedades en todos los ambientes de cultivo, excepto en la localidad porvenir (Tabla 4); estos resultados sugieren una elevada diversidad genética entre los genotipos estudiados, lo cual era de esperarse, dada la diversidad de origen de los materiales incluidos en el estudio. También se detectó diferencias significativas para los efectos de covarianza $(p<0,01)$ en tres de los cuatro ambientes y para el efecto de bloques $(p<0,05)$ en uno de ambientes de prueba, lo cual demuestra que la productividad de los materiales estuvo afectada por el número de plantas que llegaron a cosecha en cada unidad experimental y en cada ambiente, por lo que fue importante considerar el número de plantas por parcela como covariable del rendimiento de grano; también fue importante considerar el diseño de bloques incompletos (Tabla 4). Los coeficientes de variación observados en los análisis combinado e individual por localidad, se consideran apropiados para este tipo de variables en poblaciones de libre polinización.

Las localidades Beto Ega y Mantacra fueron los ambientes donde las variedades de maíz amiláceo encontraron las mejores condiciones para expresar su potencial productivo, alcanzando un rendimiento promedio de 4,605 y 4,527 $\mathrm{t} \mathrm{ha}^{-1}$, respectivamente, siendo la localidad Colcabamba (3,515 $\left.\mathrm{t} \mathrm{ha}^{-1}\right)$ la que obtuvo el nivel de productividad más bajo de todos los ambientes (Tabla 4). 
Tabla 3

Cuadrados medios para rendimiento de grano según el ANOVA combinado para 25 variedades de maíz amiláceo evaluadas en cuatro localidades de la provincia de Tayacaja en el ciclo de cultivo 2019 - 2020

\begin{tabular}{lcc}
\hline \multicolumn{1}{c}{ Fuente de Variación } & GL & $\begin{array}{c}\text { Rendimiento de } \\
\text { Grano }\end{array}$ \\
\hline Ambientes (Amb) & 3 & $14,611^{*}$ \\
Repeticiones/(Amb) & 8 & $2,558^{\star *}$ \\
Bloques/(Amb*Rep) & 48 & 0,619 \\
Variedades (Var) & 24 & $3,682^{* *}$ \\
Var*Amb & 72 & $1,377^{\star *}$ \\
Covarianza & 1 & $23,373^{\star *}$ \\
Error & 143 & 0,524 \\
Total & 299 & \\
& $\mathrm{CV}$ & 17,26 \\
\hline
\end{tabular}

GL y CV significan grados de libertad y coeficiente de variación, respectivamente ${ }^{*} \mathrm{y}{ }^{* *}$ indican diferencias significativas al $5 \%$ y $1 \%$, respectivamente.

\section{Tabla 4}

Cuadrados medios para rendimiento de grano según el análisis individual por localidad, para 25 variedades de maíz amiláceo evaluadas en cuatro localidades de la provincia de Tayacaja en el ciclo de cultivo 2019 - 2020

\begin{tabular}{lccccc}
\hline Fuente de Variación & GL & Beto Ega & Mantacra & Porvenir & Colcabamba \\
\hline Repeticiones (Rep) & 2 & 1,878 & $5,308^{*}$ & 0,769 & $2,181^{*}$ \\
Bloques/(Rep) & 12 & $0,794^{*}$ & 0,864 & 0,308 & 0,273 \\
Variedades (Var) & 24 & $1,615^{* *}$ & $4,678^{* *}$ & 0,667 & $1,284^{* *}$ \\
Covarianza & 1 & $11,811^{* *}$ & 0,118 & $9,814^{* *}$ & $4,589^{* *}$ \\
Error & 35 & 0,337 & 0,853 & 0,466 & 0,399 \\
Total & 74 & & & & \\
& CV & 12,60 & 20,41 & 16,56 & 17,97 \\
& Media & 4,605 & 4,527 & 4,125 & 3,515 \\
\hline
\end{tabular}

GL y CV significan grados de libertad y coeficiente de variación, respectivamente ${ }^{*} y{ }^{* *}$ indican diferencias significativas al $5 \%$ y $1 \%$, respectivamente.

Las variedades experimentales Cusqueado- $\mathrm{P}\left(\mathrm{G}_{8}\right)$ y Blanco Gigante-P $\left(G_{10}\right)$ presentaron los mayores niveles de productividad promedio, con 5,329 y $5,284 \mathrm{t} \mathrm{ha}^{-1}$, respectivamente, las cuales lograron superar significativamente $(p<0,05)$ a la media de producción de los testigos (4,831 tha-1) e incluso, al testigo con mayor productividad (Tabla 5). Estas dos variedades corresponden a maíces tipo choclero y para mote. Respecto a las variedades tipo maíces para cancha, Astilla Blanca-C $\left(G_{11}\right)$,
Carhuay-P $\left(G_{9}\right)$ y Chullpi-Q $\left(G_{6}\right)$ fueron las que mostraron los mayores niveles de productividad promedio, con 4,820, 4,750 y 4,626 t ha-1, siendo estos rendimientos estadísticamente iguales $(p<0,05)$ al promedio de las variedades testigos. Del mismo modo, los resultados demuestran que la variedad experimental Maíz MoradoPi $\left(\mathrm{G}^{20}\right)\left(4,905 \mathrm{t} \mathrm{ha}^{-1}\right)$ superó significativamente $(p<0,05)$ al promedio de rendimiento observado en la variedad INIA-601 ( $\left.T_{3}\right)\left(4,654\right.$ t ha $\left.^{-1}\right)$, utilizada 
como testigo para las variedades de maíces morados (Tabla 5). No obstante, la variedad INIA601 fue evaluada junto a otras variedades de maíz morado, utilizando 18 muestras de coronta y brácteas obtenidas de diferentes lugares de la sierra peruana, incluyendo cinco muestras provenientes de la provincia de Tayacaja, donde mostró excelentes niveles de antocianina (Tabla 6), atributo que le confiere un valor agregado, haciéndola prevalecer en el mercado de semilla de maíz morado en la zona de Cajamarca.

La comparación de los niveles de productividad promedio de las variedades locales vs las variedades testigos, efectuada a través de los contrastes ortogonales, demostró la superioridad en rendimiento $(p<0,01)$ y $(p<0,05)$ de los testigos (Tabla 7). Estos resultados demuestran la acumulación de alelos favorables para el carácter rendimiento de grano en maíz en las variedades mejoradas, sugiriendo la importancia del mejoramiento genético para desarrollar genotipos de superior desempeño agronómico y adaptado a las condiciones agroecológicas de las regiones de producción comercial. En este sentido, Carena (2005) señala lo valioso que significa el mejoramiento de germoplasma para desarrollar poblaciones élites, lo cual justifica el esfuerzo realizado por fitomejoradores y por las instituciones públicas y privadas que realizan el financiamiento de estas actividades. 
Tabla 5

Media de rendimiento y valores CP1, CP2 y CP3 en la evaluación de la IGA de 25 variedades de maíz amiláceo (ciclo de cultivo 2019 - 2020)

\begin{tabular}{lccccc}
\hline \multicolumn{1}{c}{ Variedad/Ambiente } & Código & $\begin{array}{c}\text { Rendimiento } \\
(\text { t.ha-1) }\end{array}$ & CP1 & CP2 & CP3 \\
\hline Astilla Amarilla-DH & G1 & 4,276 & $-0,143$ & 0,095 & $-0,229$ \\
Astilla Blanca-DH & G2 & 3,569 & $-0,506$ & $-0,345$ & $-0,118$ \\
Carhuay-Run & G3 & 4,221 & 0,476 & $-0,103$ & 0,760 \\
Carhuay-Q & G4 & 3,487 & $-0,096$ & 0,314 & $-0,040$ \\
Bolón-Q & G5 & 3,754 & $-0,216$ & $-0,053$ & $-0,118$ \\
Chullpi-Q & G6 & 4,626 & $-0,328$ & $-0,161$ & $-0,371$ \\
Astilla Blanca-P & G7 & 3,863 & $-0,296$ & 0,409 & $-0,101$ \\
Cusqueado-P & G8 & 5,329 & 0,559 & 0,633 & $-0,509$ \\
Carhuay-P & G9 & 4,750 & 0,335 & 0,173 & 0,356 \\
Blanco Gigante-P & G10 & 5,284 & 0,351 & 0,697 & $-0,127$ \\
Astilla Blanca-C & G11 & 4,820 & 0,572 & 0,015 & 0,210 \\
Almidón-ST & G12 & 3,873 & $-0,530$ & 0,281 & $-0,112$ \\
Astilla Blanca-ST & G13 & 3,792 & $-0,515$ & 0,388 & 0,186 \\
Chullpi-ST & G14 & 3,262 & $-0,466$ & $-0,275$ & 0,448 \\
Pistiada-Pi & G15 & 3,535 & $-0,154$ & $-0,071$ & 0,167 \\
Carhuay-Pi & G16 & 3,081 & $-0,250$ & 0,451 & 0,024 \\
Morocho Duro-Pi & G17 & 3,992 & $-0,272$ & $-0,369$ & 0,285 \\
Morocho Rojo Duro-Pi & G18 & 4,690 & $-0,218$ & $-0,598$ & $-0,005$ \\
Paru Rojo-Pi & G19 & 3,755 & $-0,429$ & $-0,368$ & $-0,067$ \\
Maíz Morado-Pi & G20 & 4,905 & 0,999 & $-0,605$ & $-0,174$ \\
Paru Occe-Pi & G21 & 3,843 & $-0,178$ & $-0,060$ & $-0,035$ \\
Huamamsara-Pi & G22 & 3,627 & $-0,106$ & 0,262 & 0,083 \\
Choclero-101 & T1 & 4,929 & 0,917 & 0,192 & 0,149 \\
Choclero-INIA-603 & T2 & 4,910 & 0,164 & 0,059 & $-0,233$ \\
INIA-601 & T3 & 4,654 & 0,332 & $-0,959$ & $-0,428$ \\
Beto & A1 & 4,450 & $-0,415$ & 1,383 & $-0,653$ \\
Mantacra & A2 & 3,979 & 1,854 & $-0,341$ & $-0,092$ \\
Porvenir & A3 & 4,703 & $-0,439$ & 0,291 & 1,147 \\
Colcabamba & & 3,640 & $-1,000$ & $-1,333$ & $-0,403$ \\
\hline & 4,193 & 0,000 & 0,000 & 0,000 \\
& & & & & \\
\hline
\end{tabular}


Tabla 6

Concentración de antocianinas en variedades de maíz morado evaluadas en diferentes localidades de la sierra peruana

\begin{tabular}{lcccccccc}
\hline & \multicolumn{4}{c}{ Coronta } & \multicolumn{5}{c}{ Brácteas } \\
\multicolumn{1}{c}{ Variedad } & $\begin{array}{c}\mathrm{N}^{\circ} \\
\text { Muestras }\end{array}$ & $\begin{array}{c}\text { Peso } \\
(\mathrm{g})\end{array}$ & Absorbancia & Pureza & $\begin{array}{c}\mathrm{N}^{\circ} \\
\text { Muestras }\end{array}$ & $\begin{array}{c}\text { Peso } \\
(\mathrm{g})\end{array}$ & Absorbancia & Pureza \\
\hline INIA-601 & 18 & 0.303 & 0.661 & 5.550 & 12 & 0.332 & 0.680 & 4.590 \\
INIA-615 & 6 & 0.217 & 0.642 & 6.128 & 2 & 0.825 & 0.645 & 2.195 \\
Morado & 13 & 0.488 & 0.668 & 4.592 & 6 & 0.734 & 1.690 & 1.868 \\
Mejorado & 6 & 0.402 & 0.655 & 4.332 & 3 & 0.484 & 0.650 & 2.990 \\
UNC-47 & 6 & & & & & & &
\end{tabular}

\section{Tabla 7}

Cuadrados medios para rendimiento de grano de los contrastes ortogonales realizados para comparar los niveles de productividad promedio de las variedades locales vs las variedades testigos y rendimiento medio por tipo de variedad.

\begin{tabular}{|c|c|c|c|c|}
\hline Contrastes ortogonales & $\begin{array}{l}\text { Grados de } \\
\text { Libertad }\end{array}$ & $\begin{array}{l}\text { Cuadrados } \\
\text { medios }\end{array}$ & $\begin{array}{c}\text { Tipo de } \\
\text { variedades }\end{array}$ & $\begin{array}{c}\text { Rendimiento } \\
(\mathrm{t} \mathrm{ha-1})\end{array}$ \\
\hline Locales Vs $\mathrm{T}_{1}$ & 1 & $5.482^{* *}$ & Locales & 4,106 \\
\hline Locales Vs $\mathrm{T}_{2}$ & 1 & $5.794^{* *}$ & Testigos & 4,831 \\
\hline Locales Vs $\mathrm{T}_{3}$ & 1 & $2.578^{\star}$ & $\mathrm{T}_{1}$ & 4,929 \\
\hline Locales Vs Testigos & 1 & $10.713^{\star \star}$ & $\mathrm{T}_{2}$ & 4,910 \\
\hline Error & 143 & 0.524 & $\mathrm{~T}_{3}$ & 4,654 \\
\hline
\end{tabular}

${ }^{*} \mathrm{y}$ ** indican diferencias significativas al $5 \%$ y $1 \%$, respectivamente, para el contraste de las medias."

\section{Análisis AMMI}

El análisis de los efectos principales aditivos e interacciones multiplicativas AMMI indicó que los dos primeros ejes fueron estadísticamente significativos $(p<0,01)$ y explicaron alrededor del $92 \%$ de la interacción genotipo x ambiente (IGA), en donde el CP-1 por sí solo explicó cerca del 57 $\%$ de la variación debida a la IGA. (Tabla 8). De esta manera, se observa que una alta proporción de la variación correspondiente a la IGA fue explicada por el modelo AMMI-1 con cerca del $70 \%$ de los grados de libertad de la interacción, generando un residual que apenas representó menos de $8 \%$ del efecto de la IGA, el cual resultó estadísticamente no significativo $(p>0,05)$. Los resultados, permiten inferir que el modelo fue apropiado para explicar la IGA en el conjunto de genotipos estudiados, a pesar del poco número de ambientes utilizados en el estudio. En trabajos previos también se ha demostrado la efectividad del modelo AMMI para evaluar la IGA, la estabilidad del rendimiento y el potencial agronómico con diferentes cultivares de maíz tropicales (Crossa et al., 1990; García et al., 2020; Khamphasan et al., 2018).

El modelo AMMI determinó que las variedades Astilla Amarilla-DH $\left(G_{1}\right)$, Morocho Rojo DuroPi $\left(G_{18}\right)$ y Choclero-INIA-603 $\left(T_{2}\right)$ presentaron niveles de productividad promedio superiores a la media general y elevada estabilidad a través 
de los ambientes de evaluación (Figura 1). En la Figura 1 además se observa que las variedades Cusqueado- $P\left(G_{8}\right)$ y Blanco Gigante- $P\left(G_{10}\right)$, dos materiales con tipo de grano para choclo y mote, mostraron los mayores niveles de productividad promedio, superando significativamente $(p<0,05)$ a las variedades testigos; sin embargo, su mejor desempeño productivo lo alcanzaron en la localidad Mantacra $\left(\mathrm{A}_{2}\right)$. Del mismo modo, las variedades Maíz Morado-Pi $\left(G_{20}\right)$, Astilla Blanca-C $\left(G_{11}\right)$ y Carhuay- $P\left(G_{9}\right)$ presentaron rendimientos estadísticamente $(p<0,05)$ superiores a la media general y estadísticamente $(p<0,05)$ iguales a las variedades testigo Choclero-101 e INIA-601; sin embargo, de acuerdo al modelo AMMI, el mejor desempeño de estos materiales estuvo en la localidad de Mantacra. La variedad Chullpi-Q $\left(\mathrm{G}_{6}\right)$ también presentó niveles medios de rendimientos estadísticamente $(p<0,05)$ superiores a la media general, pero sus mayores productividades la obtuvieron en las localidades Beto Ega y Porvenir. De esta manera, se puede apreciar la ventaja del modelo AMMI en facilitar determinar los genotipos con rendimientos medios superiores y de alta estabilidad y aquellos materiales con rendimientos medios superiores, pero con adaptación específica, tal como lo proponen Crossa (1990) y García et al. (2020).

Dentro del grupo de variedades incluidas en el estudio, seis de ellas Paru Occe-Pi $\left(G_{21}\right)$, Bolón-Q $\left(G_{5}\right)$, Huamansara-Pi $\left(G_{22}\right)$, Pistiada-Pi $\left(G_{15}\right)$, Carhuay-Q $\left(G_{4}\right)$ y Carhuay-Pi $\left(G_{16}\right)$ ] resultaron con alta estabilidad fenotípica para el rendimiento de grano. Sin embargo, sus niveles de productividad promedio estuvieron estadísticamente $(p<0,05)$ por debajo de la media general (Figura 1).

Tabla 8

Cuadrados medios del análisis de varianza combinado con efectos lineales y multiplicativos del modelo AMMI, para el rendimiento de grano de $\mathbf{2 5}$ variedades de maíz amiláceo evaluadas en 4 ambientes de la provincia de Tayacaja, en el ciclo de cultivo 2019-2020

\begin{tabular}{|c|c|c|c|c|}
\hline Fuente de Variación & $\mathrm{GL}$ & $\begin{array}{l}\text { Cuadrados } \\
\text { medios }\end{array}$ & $\begin{array}{l}\text { Suma de Cuadrados } \\
\text { AMMI }\end{array}$ & $\begin{array}{c}\text { Acumulado } \\
(\%)\end{array}$ \\
\hline Ambientes (Amb) & 3 & $14.611^{*}$ & & \\
\hline $\begin{array}{l}\text { Repeticiones/Amb } \\
\text { (Rep/Amb) }\end{array}$ & 8 & $2.558^{\star \star}$ & & \\
\hline Bloques /(Rep/Amb) & 48 & 0.619 & & \\
\hline Variedades (Var.) & 24 & $3.682^{\star \star}$ & & \\
\hline $\mathrm{Amb}^{\star}$ Var & 72 & $1.377^{\star \star}$ & & \\
\hline $\mathrm{CP}_{1}$ & 26 & $2,163 * *$ & 56,70 & 56.70 \\
\hline $\mathrm{CP}_{2}$ & 24 & $1,474 * *$ & 35,67 & 92,37 \\
\hline Residual & 22 & 0,345 & 7,63 & 100,00 \\
\hline Error & 144 & 0,524 & & \\
\hline
\end{tabular}




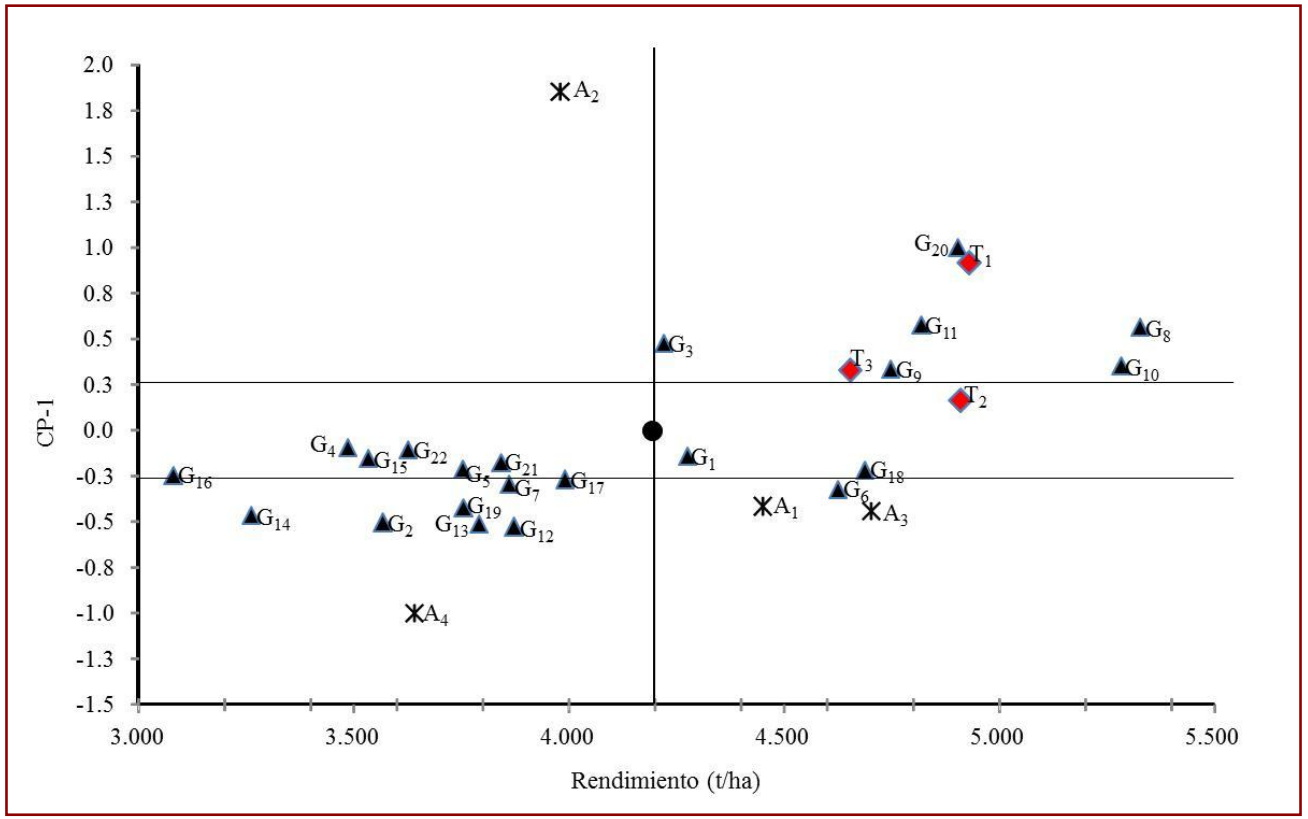

Figura 1. Modelo AMMI del comportamiento de 25 variedades (G y T) de maíz amiláceo evaluadas en cuatro ambientes (A) de la provincia de Tayacaja, Perú (ciclo de cultivo 2019-2020)

San Vicente et al., 2005; García et al., 2009 y Andrés-Meza et al., 2014 utilizaron el modelo AMMI para evaluar el desempeño agronómico de diversos cultivares de maíz y en varios ambientes tropicales y también lograron determinar genotipos con elevada estabilidad para el rendimiento de grano, pero con niveles de productividad promedio por debajo de la media general. Becker (1981) clasifica a este tipo de estabilidad como estabilidad biológica, la cual se considera de poca utilidad en la agricultura, debido a que los agricultores demandan cultivares que posean rendimientos consistentes a través los ambientes, pero que también sean capaces de responder de manera favorable a condiciones de ambientes sin estreses, es decir, cultivares con estabilidad agronómica, conforme a lo señalado por García et al. (2009).

\section{CONCLUSIONES}

- La IGA resultó altamente significativa y explicó alrededor del 33 \% de la variación fenotípica del rendimiento.

- El modelo AMMI explicó alrededor del $92 \%$ de la variación debida a la IGA, en donde los dos primeros ejes concentraron toda esta variación y permitió identificar variedades con adaptación específica y otras con amplia adaptación a los ambientes de prueba.

- Las variedades Astilla Amarilla-DH (G1), Morocho Rojo Duro-Pi (G18) y Choclero-INIA-603 (T2) resultaron con elevada productividad y estabilidad a través de los ambientes de evaluación.

- El modelo también logró determinar materiales con elevada productividad y con adaptación específica a los ambientes de prueba.

- Los resultados sugieren que el modelo AMMI fue apropiado para evaluar la IGA y para identificar las mejores variedades para cada ambiente de prueba.

\section{AGRADECIMIENTOS}

Los autores expresan su agradecimiento al Fondo de Desarrollo Socioeconómico de Camisea (FOCAM) por el financiamiento suministrado para llevar a cabo esta investigación y a los productores agrícolas Adalberto Ega, Clímaco Culcapusa llizarbe, Roberto Palomino Espinoza y al Instituto Tecnológico de Colcabamba, por el apoyo recibido, al poner a disposición sus predios para el desarrollo de los experimentos. 


\section{REFERENCIAS BIBLIOGRAFICAS}

Admassu, S., Nigussie, M. \& Zelleke, H. (2008). Genotype-environment interaction and stability analysis for grain yield of maize (Zea mays L.). Ethiopia. Asian Journal of Plant Sciences, 7, 163-169. doi: 10.3923 / ajps.2008.163.169

Adnan, A. A., Diels, J., Jibrin, J. M., Kamara, A. Y., Shaibu, A. S., Craufurd, P. \& Menkir, A. (2020). CERES-Maize model for simulating genotype-by-environment interaction of maize and its stability in the dry and wet savannas of Nigeria. Field Crops Research, 253. doi:https://doi.org/10.1016/j. fcr.2020.107826

Andrés-Meza, P., Sierra-Macías, M., MejíaContreras, J. A., Molina-Galán, J. D., Espinosa-Calderón, A., Gómez-Montiel, N. O., Valdivia-Bernal, R. (2014). Genotypeenvironment interaction in tropical maize varieties developed for the tropical region of Veracruz, Mexico. Interciencia, 39(3): 180-184. Disponible en: https:// www.redalyc.org/pdf/339/Resumenes/ Resumen_33930206007_1.pdf

Becker, H. C. (1981). Correlations among some statistical measures of phenotypic stability. Euphytica, 30:835-840. doi: https://doi. org/10.1007/BF00038812

Blum, A. (1988). Plant breeding for stress environments. Boca Raton, FL, USA: CRC Press.

Bowman, J. C. (1972). Genotype x environment interactions. Ann. Genet. Sel. Anim., 4(1), 117-123. doi: 10.1186 / 1297-9686-4-1-117

Chimonyo, V. G. P., Mutengwa, C. S. \& Chiduza, C. (2014). Genotype $\times$ environment interactions and yield stability of stresstolerant open-pollinated maize varieties in the Eastern Cape province, South Africa. South African Journal of Plant and Soil, $31(2), 61-68$. doi: https://doi.org/10.1080/02571862.2014 .868048

Chura-Chuquija, J. \& Huanuqueño-Coca, E. H. (2015). Comportamiento de ocho poblaciones de maíz amarillo (Zea mays L.) en cruzas con un probador. Anales Científicos, 76(1), 78-86. doi: http://dx.doi. org/10.21704/ac.v76i1.767

Crossa, J. (1990). Statistical analysis of multi location trials. Academic Press, Advances in Agronomy, 44, 55-85. doi: https://doi. org/10.1016/S0065-2113(08)60818-4

Crossa, J., Cornelius, P. \& Vargas, M. (2000). Modelos Estadísticos Multiplicativos para el Análisis de la Interacción Genotipo x Ambiente. México D. F., México: CIMMYT Library.

Crossa, J., H. G. Gauch \& Zobel, R. W. (1990). Additive main effects and multiplicative interaction analysis of two international maize cultivar trials. Crop Sci., 30, 493500. doi: https://doi.org/10.2135/ cropsci1990.0011183X003000030003x

Falcon, C. M., Kaeppler, S. M., Spalding, E. P., Miller, N. D., Haase, N., AlKhalifah, N. \& Leon, N. d. (2020). Relative utility of agronomic, phenological, and morphological traits for assessing genotype-by-environment interaction in maize inbreds. Crop Science, 60(1), 62-81. doi: 10.1002/csc2.20035.

Gabriel, K. R. (1971). The biplot graphic display of matrices with application to principal components analysis. Biometrika, 58, 453-467. doi: https://doi.org/10.1093/ biomet/58.3.453

García, P. J., Cabrera, S., Pérez, A., Silva, R., Álvarez, R., Marín, C., Monasterio, P. P. \& Santella, M. (2009). Estabilidad del rendimiento y potencial agronómico de cultivares de maíz de endospermo normal y QPM en zonas agroecológicas de Venezuela. Agronomía 
Trop, 59(4), 433-443. Disponible en: http:// ve.scielo.org/scielo.php?script $=$ sci arttext\&pid=S0002-192X2009000400008\& ng=es\&tlng=es.

García, P. J., Pérez, A. A., Silva, R. J., Álvarez, R. M., Pedro, M. P. \& Taramona, L. A. (2020). Evaluación del potencial agronómico dehíbridos de maíz amarillo basado en el análisis GGE biplot y el modelo AMMI. Bioagro, 32(2), 95-106. Disponible en: https://revistas.uclave.org/index.php/ bioagro/article/view/2693/1679

Gauch, H. G. \& Zobel, R. W. (1988). Predictive and postdictive success of statistical analysis of yield trials. . Theoretical and Applied Genetics, 76, 1-10. doi: https://doi. org/10.1007/BF00288824

Gauch, H. G. \& Zobel., R. W. (1996). AMMI analysis of yield trials. In M. S. Kang \& H. G. Gauch (Eds.), Genotype-by-Environment interaction (pp. 85-122). Boca Ratón. USA CRC Press. doi: http://dx.doi.org/10.1201/9781420049374. ch4

Khamphasan, P., Lomthaisong, K., Harakotr, B., Ketthaisong, D., Scott, M. P., Lertrat, K. \& Suriharn, B. (2018). Genotypic Variation in Anthocyanins, Phenolic Compounds, and Antioxidant Activity in Cob and Husk of Purple Field Corn. Agronomy, 8(11), 271. https://www.mdpi.com/20734395/8/11/271/htm doi:https://doi. org/10.3390/agronomy8110271

Li, P., Fan, Y., Yin, S., Wang, Y., Wang, H., Xu, Y. \& $\mathrm{Xu}, \quad$ C. (2020). Multi-environment QTL mapping of crown root traits in a maize RIL population. The Crop Journal, 8645 - 654 . doi: https://doi.org/10.1016/j.cj.2019.12.006

López-Morales, F., Chura-Chuquija, J. \& GarcíaPando, G. (2019). Interacción genotipo por ambiente del rendimiento de maíz amarillo en híbridos trilineales, Perú. Revista Mexicana de Ciencias Agrícolas, 10(4), 859 872. doi: https://doi.org/10.29312/remexca. v10i4.1696.

Mudada, N., Chitamba, J., Macheke, T. O. \& Manjeru P. (2017). Genotype $\times$ Environmental Interaction on Seed Cotton Yield and Yield Components. Open Access Library Journal, 4(e3192), 1-22. doi: https://doi.org/10.4236/ oalib.1103192

Ngaboyisonga, C., Nyombayire, A., Gafishi, M. K., Nizeyimana, F., Uwera, A., Ndayishimiye, T., Karemera, F., Mutanyagwa, P., Gumisiriza, G. \& Gahakwa. (2016). Adaptability and genotype by environment interaction of maize commercial hybrid varieties from East African seed companies in Rwandan environments. Global Journal of Agricultural Research, 4(2), 32-40. Disponible en: https://www.eajournals.org/wp-content/ uploads/Adaptability-and-genotype-byenvironment-interaction-of-maize.pdf

Paz, N. E., Martínez-Sánchez, J., Flores, R. A., Iñiguez, P. C., Ramírez-Córdova, A. L. \& Aparicio, Y. V. (2018). Rendimiento y Estabilidad de Híbridos Experimentales de Maíz Cultivados en el Centro de Chiapas, México. Revista Mexicana de Agroecosistemas, 5(2), 88 - 97. Disponible en: https://www.voaxaca.tecnm.mx/revista/ docs/RMAE\%20vol\%205_2_2018/2-RMAE25-maiz.pdf

Rusinamhodzi, L., Makumbi, D., Njeru, J. M. \& Kanampiu, F. (2020). Performance of elite maize genotypes under selected sustainable intensification options in Kenya. Field Crops Research, 249 (2020) 107738. doi: https:// doi.org/10.1016/j.fcr.2020.107738

San Vicente, F. S., Marín, C. \& Díaz, D. (2005). Estabilidad del rendimiento y potencial agronómico de híbridos de maíz de alta calidad de proteína (QPM) en Venezuela. Agronomía Trop., 55(3), 397-410. Disponible en: http:// ve.scielo.org/scielo.php?pid=S0002$192 \times 2005000300005 \&$ script $=s c i$ abstract\&tIng=en 
SAS, I. (2003). SAS/STAT 9 user's guide. SAS Inst., Cary, NC.

Setimela, P., Gasura, E., Thierfelder, C., ZamanAllah, M., Cairns, J. E. \& Boddupalli, P. M. (2018). When the going gets tough: Performance of stress tolerant maize during the 2015/16 (El Niño) and 2016/17 (La Niña) season in southern Africa. Agriculture, Ecosystems and Environment, 268, 79-89. doi: https:// doi.org/10.1016/j.agee.2018.09.006

Seyoum, S., Rachaputi, R., Fekybelu, S., Chauhan, Y. \& Prasanna, B. (2019). Exploiting genotype $x$ environment $x$ management interactions to enhance maize productivity in Ethiopia. European Journal of Agronomy, 103, 165-174. doi: https://doi.org/10.1016/j. eja.2018.12.011

Souza, A., Miranda, G., Gonzaga, M. \& Souza, L. (2009). Predicting the genetic gain in the Brazilian white maize landrace. Ciência Rural, 39, 19- 24. doi: https://doi.org/10.1590/ S0103-84782009000100004

Steel, R. \&Torrie, J. (1988). Bioestadística: Principios y procedimientos (2da ed.). México: McGraw-Hill /Interamericana.

UNAT (Universidad Nacional Autónoma de Tayacaja “Daniel Hernández Morillo"). (2018). Selección de cultivares y conservación de la identidad genética de variedades de maíz (Zea mays L.) en la provincia de Tayacaja. Proyecto aprobado según Resolución de la Comisión Organizadora Nº126-2018-COUNAT (pp. 49). Perú. Disponible en: https:// unat.edu.pe/nuestras-investigaciones/.

Vargas, H. M. \& Crossa., J. (2000). El análisis AMMI y la gráfica del biplot en 42. Retrieved from SAS. CIMMYT, INT. website: www.cimmyt. cgiar.org/biometrics

Yan, W., Kang, M., Ma, B., Woods, S. \& Cornelius, P. L. (2007). GGE Biplot vs. AMMI Analysis of Genotype-byEnvironment Data. Crop Sci., 47, 641-653. 